\title{
Histiocytic necrotising lymphadenitis in systemic lupus erythematosus
}

\author{
M D Litwin, B Kirkham, D R F Henderson, S C Milazzo
}

\begin{abstract}
Histiocytic necrotising lymphadenitis is the pathognomonic histological appearance of lymph nodes in Kikuchi's disease, a condition characterised by a brief systemic illness and lymphadenopathy.

The case is described of a young man, originally diagnosed as having Kikuchi's disease by lymph node histology, who subsequently developed systemic lupus erythematosus with symmetrical polyarthritis, Coombs' positive haemolytic anaemia and haemorrhagic pneumonitis. The case emphasises that a range of diseases is associated with histiocytic necrotising lymphadenitis, belying the unitary impression given by the term Kikuchi's disease.
\end{abstract}

Histiocytic necrotising lymphadenitis was first described in Japan in $1972,{ }^{12}$ and is now increasingly recognised in Europe and the USA. ${ }^{3}$ Its diagnosis is based on a characteristic histological appearance of 'lymphadenitis showing focal reticulum cell hyperplasia with nuclear debris and phagocytosis'. ${ }^{3}$ Recent case reports $^{4-8}$ and an editorial ${ }^{9}$ have tended to reinforce the association of histiocytic necrotising lymphadenitis with a brief illness in previously healthy women which has a benign, uncomplicated prognosis. So confidently has this pathological lymphadenopathy been assumed to represent a discrete clinical entity that it has received an eponymous name, 'Kikuchi's disease', after one of the first Japanese pathologists to describe it. Although it is important that doctors recognise the characteristic, self limiting presentations of histiocytic necrotising lymphadenitis with systemic illness and lymphadenopathy, they need also to appreciate that some patients with the same pathological diagnosis go on to develop progressive and severe disorders. One review has briefly described the development of systemic lupus erythematosus (SLE) after an initial diagnosis of histiocytic necrotising lymphadenitis. ${ }^{3}$ We report a florid case of SLE, presenting initially as histiocytic necrotising lymphadenitis.

Queen Elizabeth
Hospital,
28 Woodville Road,
Woodville South,
South Australia,
Australia 5011
M D Litwin
B Kirkham
D R F Henderson
S C Milazzo
Correspondence to:
Dr Kirkham.
Accepted for publication
6 August 1991

Queen Elizabeth

Woodville South, South Australia,

Australia 501

M D Litwin

B Kirkham

Dr Kirkham.

Accepted for publication weight loss of $6 \mathrm{~kg}$ over a period of one month. Both his mother and grandmother had Hodgkin's disease. His past history included chronic asthma and eczema, and repair of a duplex oesophagus. Physical examination showed marked bilateral cervical lymphadenopathy. The nodes were firm and not tender, with the largest measuring $6 \times 6 \mathrm{~cm}$. Smaller bilateral axillary and inguinal nodes were also noted.

Investigations showed a mild lymphopenia of $0.8 \times 10^{9} / 1$ (normal $1.3-3.4 \times 10^{9} / 1$ ) with occasional atypical lymphocytes, a microcytosis of $69 \mathrm{fl}$ (normal 81-98 fl) due to a $\beta$ thalassaemia trait, with haemoglobin $135 \mathrm{~g} / \mathrm{l}$ (normal 130-165 $\mathrm{g} / \mathrm{l}$ ) and an erythrocyte sedimentation rate of 18 $\mathrm{mm} /$ hour. Viral, toxoplasma, and HIV serology were all negative and a chest radiograph was normal.

Histology of a cervical node obtained by open biopsy showed scattered areas of necrosis, mainly in the interfollicular areas, surrounded by large numbers of proliferating, immature, mononuclear cells, some of which were atypical and mitotically active. There were also scattered foamy macrophages, lymphocytes, and a few polymorphs. Some lymphoid follicles could still be seen but the germinal centres were not prominent. The diagnosis made was histiocytic necrotising lymphadenitis, or Kikuchi's disease.

The initial symptoms, other than lethargy, improved spontaneously and the lymphadenopathy became less prominent. Two months after presentation lymphopenia persisted and the erythrocyte sedimentation rate was 35 $\mathrm{mm} /$ hour. Total haemolytic complement was $20 \%$ (normal $90-96 \%$ ) with a low C3 of $0.34 \mathrm{~g} / 1$ (normal $0.48-1.35 \mathrm{~g} / \mathrm{l}$ ), and a low C4 of $0.09 \mathrm{~g} / \mathrm{l}$ (normal 0.15-0.48).

Seven months after presentation he was less well and had developed a symmetrical, generalised polyarthritis with early morning stiffness lasting one hour and reported dyspnoea with small intermittent haemoptyses. Synovitis was present in most joints, bilateral basal pulmonary crepitations were audible, he was clinically anaemic, and generalised lymphadenopathy persisted.

Investigations were now consistent with an IgG/C3d Coombs' positive haemolytic anaemia, with haemoglobin $65 \mathrm{~g} / \mathrm{l}$, reticulocytes $6 \%$ (normal $0 \cdot 2-2 \cdot 0 \%$ ), erythrocyte sedimentation rate $96 \mathrm{~mm} /$ hour, and total haemolytic complement $<20 \%$. Antinuclear antibody was positive at a titre of $1 / 2560$, homogeneous pattern, with antibody to double stranded DNA $140 \mathrm{IU} / \mathrm{ml}$ (normal 0-7 IU $/ \mathrm{ml}$ ). A chest radiograph showed cardiomegaly and peribronchial thickening, but a computed tomography chest scan also showed patchy left upper lobe consolidation. Ventilation perfusion pulmonary scintigraphy was normal. Respiratory function tests showed a restrictive pattern with a forced vital capacity $67 \%$ of the predicted value. The carbon mon- 
oxide transfer factor $\left(\mathrm{K}_{\mathrm{CO}}\right)$ was $120 \%$ of the predicted value. Bronchoscopy without biopsy or lavage was normal.

A diagnosis of SLE was made and treatment with prednisolone, $30 \mathrm{mg} /$ day, was begun. His arthropathy, lymphadenopathy, dyspnoea, and malaise rapidly improved. The anaemia was resistant, despite an increase of the dose of prednisolone to $100 \mathrm{mg} /$ day. Blood loss was suspected and an active duodenal ulcer was found at endoscopy. The haemoglobin increased steadily after ranitidine $\left(\mathrm{H}_{2}\right.$ receptor antagonist $)$ and iron replacement were added.

Twelve months later he remained well apart from mild, non-limiting dyspnoea during strenuous sporting activities, and small streaking haemoptyses on most days. His ongoing treatment was hydrochloroquine $400 \mathrm{mg} /$ day and prednisolone $10 \mathrm{mg}$ every other day. Chest radiographs at this time showed a transient right sided pulmonary infiltrate clearing partially within one week. The cardiac profile was normal. His forced vital capacity was $74 \%$ of the predicted value and the $K_{C O} 84 \%$ of the predicted value. Maximum inspiratory and expiratory airway pressures were normal, suggesting unimpaired respiratory muscle strength. The erythrocyte sedimentation rate was $9 \mathrm{~mm} /$ hour, the antinuclear antibody titre was $1 / 640$ with antibodies to double stranded DNA $17 \mathrm{IU} / \mathrm{ml}$; C3 was normal whereas C4 was marginally reduced at $0.13 \mathrm{~g} / \mathrm{l}$. A transbronchial lung biopsy showed pulmonary haemorrhage with widened septa which stained for IgG, consistent with lupus pneumonitis.

High dose prednisolone, $60 \mathrm{mg} / \mathrm{day}$, was started again. After four months treatment his chest radiograph was clear and the dyspnoea and haemoptysis had improved, though they had not entirely abated. A second lung biopsy showed decreased but continuing fresh haemorrhage and decreased septal IgG. Azathioprine $100 \mathrm{mg} /$ day was introduced. Four months later he still has haemoptyses of minimal volume approximately once a week.

\section{Discussion}

The patient had a triphasic illness beginning with a lymphoma-like cervical lymphadenopathy, followed by a florid multisystemic lupus which resolved to leave a chronic minimally symptomatic pneumonitis. The first two phases show an association between the histological diagnosis of histiocytic necrotising lymphadenitis and SLE, whereas the third phase questions how intensively we treat isolated, symptomatically mild, yet evident pulmonary abnormalities in chronic SLE. Published work on histiocytic necrotising lymphadenitis, which is written mainly by pathologists, not only

Number of antibody secreting cells in peripheral blood (expressed per $10^{3}$ mononuclear cells for $\operatorname{IgG}, \operatorname{IgA}$, and $\operatorname{IgM})^{13}$

\begin{tabular}{|c|c|c|c|}
\hline $\begin{array}{l}\text { Time after presentation } \\
\text { (months) }\end{array}$ & $\begin{array}{l}\operatorname{Ig} G \\
\text { (reference }<0.5)\end{array}$ & $\begin{array}{l}\operatorname{IgA} \\
(\text { reference }<1 \cdot 6)\end{array}$ & $\begin{array}{l}\text { IgM } \\
\text { (reference }<0.3)\end{array}$ \\
\hline $\begin{array}{r}0 \\
2 \\
7 \\
19\end{array}$ & $\begin{array}{c}4 \cdot 39 \\
31 \cdot 2 \\
9 \cdot 0 \\
0.69\end{array}$ & $\begin{array}{l}2 \cdot 71 \\
4 \cdot 7 \\
2 \cdot 2 \\
0 \cdot 91\end{array}$ & $\begin{array}{l}0.55 \\
1.3 \\
0.7 \\
0.26\end{array}$ \\
\hline
\end{tabular}

confirms its distinction from malignancy, but emphasises that histiocytic necrotising lymphadenitis is a separate entity associated with the benign clinical features of Kikuchi's disease. For example, Pileri et al state ${ }^{10}$. . . 'Clinically, the lesion often appears as lymphadenopathy in the neck; the enlarged nodes are painful. Manifestations in sites other than the cervical region and generalised lymphadenopathy are less common. Fever and leukopenia are observed frequently. The prognosis is always excellent, and many patients recover without treatment ....'

The diagnosis of Kikuchi's disease was reconsidered as multiple features of SLE became obvious. Support for our contention that the original illness was SLE came subsequently from a positive antinuclear antibody titre of $1 / 640$, with antibodies to double stranded DNA $13 \mathrm{IU} / \mathrm{ml}$, results available retrospectively from serum samples saved from the first presentation.

Generalised lymphadenopathy of the size seen in this patient is rare as a presenting feature of SLE. Nodal size is usually not more than three to four centimetres. ${ }^{11}$ Dubois found cervical lymphadenopathy to be the presenting feature of SLE in $1.7 \%$ of patients, and generalised adenopathy in $0 \cdot 5-2 \cdot 0 \% .^{11}$ of established cases $50 \%$ had generalised lymphadenopathy. The histology of nodes in SLE is variable $^{12}$ and can overlap those changes seen in histiocytic necrotising lymphadenitis. ${ }^{3}$

B lymphocyte activation was assessed in our patient by assaying the number of peripheral blood cells secreting IgG, IgA, and IgM, at various times during his illness (see table). This paralleled other parameters of disease activity. Increased numbers of antibody secreting cells are a characteristic feature in active SLE $^{13}$ but findings in histiocytic necrotising lymphadenitis have not previously been reported.

Despite his overall improvement, mild dyspnoea and haemoptysis persisted. Fleeting pulmonary infiltrates and increased $K_{C O}$ values suggested pulmonary haemorrhage, which was confirmed. Reports of alveolar haemorrhage in SLE describe acute, life threatening events usually in the context of glomerulonephritis and hypocomplementaemia, unlike the relatively asymptomatic nature of this problem. ${ }^{14-16} \mathrm{High}$ doses of steroids and immunosuppressive drugs were given empirically because of the perceived threat to his lungs. There is, however, a lack of prognostic and management data about this aspect of SLE.

The association of SLE with histiocytic necrotising lymphadenitis has been suggested in one previous review, but the five patients included were neither as florid as this patient, nor were they fully documented clinically. Medeiros et al ${ }^{17}$ reported established SLE preceding the appearance of histiocytic necrotising lymphadenitis-type lymphadenopathy. Ohta et al ${ }^{18}$ and Lyberatos ${ }^{19}$ describe patients in whom the typical lymphadenopathy was among the presenting features of Still's disease.

The clinical spectrum of associations of histiocytic necrotising lymphadenitis is wide and ranges from otherwise asymptomatic 
adenopathy, pyrexia of unknown origin, nonspecific rashes, myalgias and arthralgias, mesenteric adenitis, Still's disease, and SLE, through to death. ${ }^{20}$ This spectrum of associations of histiocytic necrotising lymphadenitis belies the unitary impression given by the term 'Kikuchi's disease'. We believe it would be best for doctors to avoid using the latter name as this suggests a discrete, benign, clinical entity, and hence reduces awareness of more serious diseases that may present with identical pathological and clinical features.

The aetiology of both histiocytic necrotising lymphadenitis and SLE is unclear. Reports of patients initially diagnosed as having histiocytic necrotising lymphadenitis who subsequently developed SLE and adult Still's disease suggest that these conditions have similar causative factors. Although white patients are well represented in case series of histiocytic necrotising lymphadenitis, a large number of case reports have been of Asian patients. Our patient was of Greek descent and had lived in Australia most of his life, but the repair of his duplex oesophagus at the age of 13 years was performed in Hong Kong, from where 10 cases of histiocytic necrotising lymphadenitis have been reported. ${ }^{2021}$ It is interesting to speculate if an operation in this country with a high occurrence of histiocytic necrotising lymphadenitis could have been the mode of transmission of an infectious agent, which has subsequently predisposed to both histiocytic necrotising lymphadenitis and SLE.

The authors gratefully acknowledge Professor A G Wangel, and Drs D A Campbell, B Kuss, H Costi, and J Barbara for reviewing this report.

1 Kikuchi M. Lymphadenitis showing focal reticulum cell hyperplasia with nuclear debris and phagocytosis. Nippon Ketsueki Gakkai Zasshi 1972; 35: 379-80.

2 Fujimoto Y, Kozima Y, Yamaguchi K. Cervical subacute necrotising lymphadenitis. A new clinicopathological entity. Nakika 1972; 20: 920-7.

3 Dorfman R F, Berry G Y. Kikuchi's histiocytic necrotising lymphadenitis: an analysis of 108 cases with emphasis on
differential diagnosis. Semin Diagn Pathol 1988; 5: 329-45.

4 Bailey E M, Klein N C, Cunha B A. Kikuchi's disease with liver dysfunction presenting as fever of unknown origin Lancet 1989; ii: 986.

5 Kapadia V, Robinson V A, Angus H B. Kikuchi's disease presenting as fever of unknown origin. Lancet 1989; ii: 1519.

6 Nieman R B. Diagnosis of Kikuchi's disease. Lancet 1990 335: 295 .

7 Bowness P, Dutoit S H. Kikuchi's disease as a cause of fever and cervical lymphadenopathy. F Infect 1988; 16: 310-11.

8 Rudniki C, Kessler E, Zarfati M, Turani H, Bar-Ziv Y Zahavi I. Kikuchi's necrotising lymphadenitis: a cause of fever of unknown origin and splenomegaly. Acta Haematol (Basel) 1988; 79: 99-102.

9 Anonymous. Kikuchi's disease. Lancet 1990; 335: 1563.

10 Pileri S, Kikuchi M, Helbron D, Lennert K. Histiocytic necrotising lymphadenitis without granulocytic infiltration. necrotising lymphadenitis without gran
Virchows Arch [A] 1982; 395: $257-71$.

11 Dubois E L. The clinical picture of systemic lupus erythematosus. In: Dubois E L, ed. Lupus erythematosus, 2nd ed. Los Angeles: University of Southern California Press, 1974: 238-343.

12 Cruickshank B. The basic pattern of tissue damage and pathology of systemic lupus erythematosus. In: Dubois E L, ed. Lupus erythematosus 2nd ed. Los Angeles: University of Southern California Press, 1974: 36-38, 57-58.

13 Wangel A G, Milton A, Egan J B. Spontaneous plaque forming cells in the peripheral blood of patients with systemic lupus erythematosus. Clin Exp Immunol 1982; 49: system.

14 Leatherman J W, Davies S F, Hoidal J R. Alveolar hemorrhage syndromes. Medicine (Baltimore) 1984; 63: 343-61.

15 Myers J L, Katzenstein A A. Microangitis in lupus-induced pulmonary hemorrhage. Am $\mathcal{F}$ Clin Pathol 1986; 85: 552-6.

16 Eagen JW W Mem G R, Schwartz M M, Lewis E J. Pulmonary hemorrhage in systemic lupus erythematosus. Medicine (Baltimore) 1978 57: 545-60.

17 Medeiros L J, Kaynor B, Harris N L. Lupus lymphadenitis: report of a case with immunohistologic studies on frozen sections. Hum Pathol 1989; 20: 295-9.

18 Ohta A, Matsumoto Y, Ohta T, Kaneoka H, Yamaguchi M. Still's disease associated with necrotising lymphadenitis (Kikuchi's disease): report of 3 cases. $\mathcal{F}$ R heumatol 1988; 15: 981-3.

19 Lyberatos C. Two more cases of Still's and Kikuchi's. $f$ Rheumatol 1990; 17: 568-9.

20 Chan J K C, Wong Kong-Chiu, Ng Chi-Sing. A fatal case of Chan J K C, Wong Kong-Chiu, Ng Chi-Sing. A fatal case of

21 Chan J K C, Saw D. HNL; a clinicopathologic study of 9 cases. Pathology 1986; 18: 22-8. 